\title{
A queueing model for evaluating traffic police infraction registration system
}

\begin{abstract}
This paper is a consequence of a main project, the project is a novel experiment which provides "live" traffic ticket issuing and registration. One of the main police requirements is information systems to provide information for police anywhere and anytime. We made some efforts to improve police performance with new solution. Advanced of civilian wireless network infrastructure made the mobile devices increasingly popular. Mobile equipments are widely used for communication but lacking of security makes it unsuitable for transmitting the confidential data. In this paper, we discuss requirements of the traffic police data center firstly, and then this paper is produce a generally applicable queuing model that abstracts all Traffic Police Data Center components, in a queuing model of a Traffic Police Data Center, the customers are officers, the servers are located on Traffic Police Data Center (TPDC) and queues are populated by traffic tickets that wait for the services. Finally, we focus on the resources monitoring.
\end{abstract}

